\title{
ICTs for Emotional and Social Skills Development for Children with ADHD and ASD Co-existence
}

\author{
https://doi.org/10.3991/ijet.v14i05.9430 \\ Lizeta N. Bakola $\left({ }^{\varpi}\right)$, Nikolaos D. Rizos, Athanasios S. Drigas \\ National Center for Scientific Research "Demokritos", Agia Paraskevi, Greece \\ lizeta79@gmail.com
}

\begin{abstract}
ADHD and autism, also called Autism Spectrum Disorder (ASD), according to the DSM-5, are both two of the most common neurodevelopmental disorders in childhood although they can continue into adolescence. ADHD is characterized by inattention, hyperactivity and impulsivity and Autism by problems with social interactions and stereotyped (repetitive or ritualistic) behaviors. Many studies have proved that the two conditions can occur together and furthermore, that children with ADHD and or autism disorder can have or develop a co-morbid psychiatric disorder. In this paper we review research on the presentation of these two behavioral and emotional disorders as ADHD and Autism ,their common symptoms and characteristics as well as the significant and major contribution of ICT's who shows magnificent promise to improve the quality of life for children with ADHD and ASD.
\end{abstract}

Keywords-Emotional skills; social skills; Autism Spectrum Disorder; ADHD

\section{Introduction}

ADHD and Autism, also called ASD, are two of the most common neurodevelopmental disorders affecting both children and adults, according to the Diagnostic and Statistical Manual of Mental Disorders, Fifth Edition (DSM-5). ADHD is described as "persistent" or on-going pattern of inattention and /or hyperactivity- impulsivity that gets in the way of daily life or typical development. ASD is the name for a group of developmental disorders. Autism Spectrum Disorder includes a wide range, "a spectrum" of symptoms and disabilities in communication and social skills and stereotypical behaviors as well. These two disorders are related in several ways. Although ADHD is not on the Autism Spectrum Disorder, they seem to have some of the same symptoms. For example, in both conditions, children have difficulties in paying attention, troubles with social interactions, exhibit hyperaction, present difficulties in reading social cues and have meltdowns, but for different reasons. Moreover, it is found that having one of these conditions also increases the likelihood of having the other. According to the experts, these two disorders are also related by sharing genetic risks and may have a similar underlying basis as well as a person with autism has an increased chance of having a close relative with ADHD or 
another developmental or a co-morbid psychiatric disorder. The last few years the consideration of experts about how autism and ADHD are related has changed. According to the DSM-IV, a person couldn't have autism and ADHD simultaneously. On the contrary, the DSM-V was updated in 2013 and made changes to the definition of the disorders and from now on it allows for a person to be diagnosed with both. The symptoms of ADHD and autism are difficult to be distinguished and can many times be overlapped. Furthermore, the bottom line that distinguishes ADHD from autism is the ability to intuitively comprehend the social world (Mark Berlin Oct. 27, 2016). Since autism and ADHD overlap symptoms, some of the interventions for one can be helpful for the other. In this review paper, we will try to present examples of ICTs' therapies and interventions available for children with ASD and ADHD, some of which might be able to help children with recognizing and showing emotions (ASD) and others who can help children with ADHD to regulate their attention and behavior [1], [2], [3], [4].

\section{Social Difficulties and Emotional Understanding in Children and Adolescents with ADHD and ASD: The Co-relation}

Although there are many reports pertaining to social difficulties in ADHD, little is still known because there are no many studies about the diversity of social problems, their etiology or their correlation to the disorders of social behavior, such as autism or PDD. In 2009 Rich Erika Carpenter et al conducted a research in 379 children and adolescents with ADHD by using the Child Behavior Checklist (Achenbach, 1991) to investigate and improve the association with PDD risk. According to the researchers the description of Cantwell (1996) regarding to the social difficulty in ADHD is similar to the poor social functioning in PDD described as a "lack of savoir faire". It is mentioned that until then the exploration of the true overlap of PDD and ADHD was difficult and limited because of the DSM-IV in which a dual diagnosis was precluded. Nevertheless, literature from both phenotype and molecular genetic investigations was beginning to address the common characteristics of some domains of ADHD with those of PDD. The researchers, according to many studies, project all the difficulties with social interaction in children with ADHD and PDD and indicate all the common characteristics such as the risk for peer rejection, disabilities in social exchange and inflexibility. More specifically, it is reported that multiple studies of social functioning difficulties in ADHD have focused on co-morbid behaviors and ADHD symptomatology as sources of poor social functioning and those are: specifically aggression, disruptive behavior disorders and hyperactive -impulsive symptoms. Moreover it is found that $2 / 3$ of individuals with ADHD have a co-morbid psychiatric disorder such as mood disorder, anxiety, conduct disorder, or anxiety and depression together. On the other hand in PDD the social disabilities include a preference to play alone, a lack of social reciprocity, difficulty in reading non-verbal social cues, a one-sided conversational style and an impaired ability to understand the emotions, motivations and intentions of peers. Regarding to diagnostic and symptoms similarities between $\mathrm{ADHD}$ and $\mathrm{PDD}$, studies and statistics prove that children 
referred to clinics for PDD also present with significant ADHD symptoms, like inattention, hyperactivity and impulsivity. For the present study a sample of a subset of families with at least one child with ADHD who participated previously in an ADHD Genetics study was included. All families were evaluated in a two-step process, at first by using the SNAP-IV behavior rating scale as a screening tool for the presence of ADHD in children between the ages of 5 and 18 and subjects were screened for other inclusion and exclusion criteria. In addition, two basic semistructured interviews were used, the K-SADS-PL for ages 5-17 and SADS-LAR-IV for ages 18 and older. All interviews were conducted by clinical psychologists or highly trained interviewers with extensive experience in psychiatric diagnoses. Diagnoses were based on all available information, including WISC scores, CBCL, RMSEA, CFI etc. As a conclusion, it was found that there is a strong relationship between PDD risk and ADHD, but more research is needed utilizing alternative tools of assessment including genetic, brain imaging methods and neuropsychological testing to investigate etiological factors that may be shared across diagnostic classifications [5].

Moreover, according to Craig F. et al., many recent studies support several overlapping traits between Attention -Deficit/Hyperactivity Disorder (ADHD) and Autism Spectrum Disorders. In their particular study, the specialists tried to assess the common or distinguishable clinical features between ASD and ADHD for the purpose of discover possible different phenotypes that could have a clinical value. Pursuant to their article, ASD and ADHD are both childhood-onset neurodevelopmental disorders, with prevalence of $1 \%$ and $5 \%$ in the pediatric population. The DSM-IV criteria, could not allow a diagnosis of ADHD if the symptoms of inattention and hyperactivity were developed at the same time with a pervasive developmental disorder (PDD) (Craig F. et al 2015). Nevertheless, new epidemiological, clinical and neuroimaging findings have changed the thought of the ADHD exclusion criteria with the recent publication of the DSM-5 and from now on both ASD and ADHD can be diagnosed together (Craig F. et al 2015). It is referred that despite some important differences, these two disorders exhibit many similar disabilities that could complicate a differential diagnosis such as attention deficit, behavior problems, and difficulty in social skills. Craig F. et al., report a list of recent studies (on biological risk factors, neuropsychological domains, and brain imaging), that support high rates of ADHD comorbidity in children with ASD, elevated rates of autistic symptoms in children with ADHD, as well as several overlapping traits between ASD and ADHD. Taurines et al. (2012) suggest that comorbidity is caused by overlapping genetic or non-genetic biological risk factors. The family and twin studies seems to support the hypothesis of familial/genetic factors (Reiersen et al., Ronald et al. 2008) (Craig F. et al 2015). On the other hand, the specific non-genetic biological risk factors associated with overlapping between ASD and ADHD include : psychotropic medications, preterm birth, maternal pre-eclampsia, maternal autoimmune disease and maternal infection disease (Cohen et al.,2011, Croen et al. 2011, Kroger et al. 2011, Lyall et al. 2012, Taurines et al., 2012) (Craig F. et al 2015) . Considering all these data, Craig F. et al., used a sample of 181 subjects, all referred to University Hospital of Bari, in order to evaluate the common or distinctive clinical features between ASD and 
ADHD and identify possible different phenotypes. The participants were divided into four diagnostic groups: ADHD group (51 children and adolescents), ASD group (43 children and adolescents), ASD+ADHD group (31 children and adolescents that met criteria for both ASD and ADHD), and the control group. The diagnoses of these patients were all made by clinical experts according to DSM-IV-TR and were based on the developmental histories of the children, taken for clinical interviews with the parents, observations and extended neuropsychological testing of the children themselves. The criteria for the ASD patients were the typical triad of symptoms of autism: social deficits, communication impairement and rigid ritualistic interests (Craig F. et al 2015). Moreover, ADHD patients had to present the typical core symptoms of inattention, hyperactivity and impulsivity. The ASD+ADHD patients had to satisfied the requirements of DSM-IV-TR diagnostic criteria for both ASD and ADHD. IQ, emotional and behavior problems, ADHD symptoms, ASD symptoms and adaptive behaviors were investigated trough several tests such as WISC, WPPSI or LIPSR, CBC, CRS-R, SNAP-IV Rating Scale, the SCQ and VABS. According to the scientists, the results shown that the ASD+ADHD group differs from ASD or ADHD groups in some domains such as in lower IQ mean level and in a higher autistic symptoms severity. Moreover, the ASD+ADHD group seems to maintain some clinical aspects that characterize ASD or ADHD group. More specifically the inattention and hyperactivity deficit as well as some emotional and behavioral problems seems to be shared with the ADHD group, while it also shares adaptive behavior impairement with the ADHD group. In conclusion, these findings provide some new understanding of the ASD+ADHD phenotype but more research is demanded [6], [7], [8], [9].

\section{Assistive Technology for ADHD and ASD Symptoms: ICTs have Emerged as Important Tools for Social and Behavioral Difficulties}

Sabrina Schuck et al. conducted a pilot study to evaluate the feasibility and utility of iSelfControl, which is a web-based application. iSelfControl provides a platform for student and teacher evaluation thereby improving self-regulation and behavior management of children with ADHD symptoms inside the classroom. It is convenient, easily accessible, motivating and less expensive or stigmatizing compared to other applications, such as computers and other specialized devices. It can be also used in conjunction with conventional classroom management strategies, since children with ADHD receive at least $80 \%$ of their instruction in the general education classroom. iSelfControl is based on supporting three processes:

- Behavior monitoring by focusing the attention on the situation ("What am I doing right now?',)

- Behavior evaluation (“what am I supposed to be doing right now?")

- Behavior correction ("what can I do to meet my goals?'). 
Students are prompted to log in the platform every 30 minutes (this period is called 'Center') and evaluate their behavior (perceive and score themselves). They also earn points for demonstrating adaptive behaviors in domains, such as 'Following Directions", "Following Rules", "Staying on Task" and "Getting alone with others". Simultaneously, the teacher records behavioral observations for each child on a separate iPad. Afterwards, the students can compare their scores to the ratings made by their teacher. In this way, they pay attention, think about their actions and are provided with feedback on their progress. The pilot study was conducted in a classroom with students aged 9-11 years with ADHD primarily over a six-week period. The results indicated that students had the tendency to evaluate their behaviors more positively compared to their teachers-reported scores, but this is likely to happen given that they demonstrate weal self-awareness due to their difficulties. However, self-awareness gradually improved during the day and over the period of the trial. In conclusion, iSelfControl is a promising platform for self-awareness and selfregulation by suggesting evidence-based, individualized intervention strategies and encouraging children to compare their estimations to their teacher's observations [7].

Furthermore, emotional outbursts and high levels of stress are often experienced by children with ADHD symptoms. This is the reason why Tobias Sonne and Mads Moeller Jensen (2016) present Chillfish, a calming biofeedback game that helps children with ADHD control their breath and stress level. Biofeedback games use neurobiological or physiological data as input to control and affect a game and can replace traditional treatments, like medication. Different kinds of sensors and portable devices are used which detect the heart rate variability (HRV) or other signals and assist the user to lower the breathing rate. However, the special equipment, which is usually required, renders such applications not suitable enough for children. ChillFish was designed for children and combines a breathing exercise with a video game retaining the child's attention and calmness. It is based on an underwater $2 \mathrm{D}$ world where a fish collects as many starfishes as possible in 2 minutes. The movements of the fish are controlled through inhaling and exhaling via a physical LEGO fish in which electronics (RFDuino, Bluetooth 4.0) are embedded. When the player breathes into the LEGO fish, the digital fish inflates and moves to the top and the bottom of the screen. The changes in the temperature during the breathing are detected by a thermistor and determine the character's moves. A study, in which 16 adults participated, revealed the positive effects on the player's stress levels compared to other activities, such as PACMAN, relaxation exercise and casual conversations. The HRV values in the ChillFish and relaxing activities were very similar and had almost the same physiological impact on the player. Moreover, the use of respiration as a game controller, the underwater theme with visual and auditory stimuli and the less stressful point system result in the increase of players' mean HRV values, their relaxation and calmness as well [8].

Sonne, Marshall et al. conducted a follow-up study following a field study in which 13 children with ADHD and their families participated. The subjects of the study used an assistive technology, named MOBERO, in order to change family practices and establish healthy morning and bedtime routines. As it is mentioned, ADHD is a heterogeneous disorder with various consequences, two of which are bedtime 
resistance and sleep problems. Since sufficient sleep plays a crucial role in people's overall performance, a lot of research has been done in this field to help adults with improving their sleep habits; ShutEye and Lullaby are two relevant examples. As far as children are concerned, assistive technology includes TangiPlan, ChillFish, BlurtLine and CASTT. MOBERO is another mobile tool which focuses on both parents' and children's routines and consists of two modules, a bedtime and a morning one. Each module is structured on a list of icons and text and facilitates either parents or children as well to manage their time and organize their activities. Children can also be rewarded if they complete the activities in a specific timeframe. The study is divided into two phases: the baseline and the intervention period (2 weeks respectively) and a follow-up study (1 month). The assessment of parents' responses in MOBERO-Q Questionnaire suggests that their frustration and conflict level around and during morning and bedtime routines reduced and that the lowering effect of MOBERO was still sustained one month after the intervention period. The authors conclude that, despite the different way some families were affected by the mobile application, they all experienced positive changes in their practices related to their children's sleep routines, thereby making future research on assistive technologies for children with ADHD promising [9].

Sonne et al. present a design framework for ADHD assistive technologies, both the existing ones and future research efforts. The framework is based on empirical studies, ADHD research and work on technologies, it couples ADHD difficulties to technological applications and suggests strategies for their development. According to Faraone et al. (2015), the challenges a patient with ADHD faces could be grouped in:

- Social disabilities

- Academic and occupational failure

- Health problems and psychiatric co-morbidities

- Psychological dysfunction

- Risky behaviors

Similarly, the assistive technologies can be categorized in 3 groups:

- MIIS Technologies, which means manually interacted with information and services

- AES Technologies, referring to automatically executing services based on in-situ analysis of context information

- CCD Technologies, which capture contextual data for later retrieval.

More specifically, the first category includes technologies that are manually triggered or interacted with, either for assistance in specific situations or for training. Such examples are MOBERO, ChillFish, TangiPlan and TimeTimer. The second group is services which analyze captured data providing assistance to the user (Parent Guardian, CASTT, BlurtLine). As far as the third approach is concerned, CCD, it covers context aware technologies which collect data for services for research and services for personal reflection. This category still remains unexplorable for the ADHD domain, but examples of CCD Technologies from related domains, such as 
MONARCA and Lullaby, indicate that significant potential is held for ADHD as well. It is also highlighted that the design framework can be used to identify new opportunities for future research on ADHD assistive technologies by expanding existing research as well as creating novel research approaches. In the end, the researchers suggest a set of strategies to maximize the impact of research on ADHD technologies and, specifically, providing structure to facilitate activities, minimizing distractions, encouraging praise and rewards and, finally, integrating and reporting standardized ADHD measures [10].

B. O. Ploog, A. Scharf et al (2013) made a review of studies and research that had been conducted in how computer- assisted technology (CAT) can be used in Autism Spectrum Disorder to improve social, communicative and language development of individuals. The literature is divided into four main areas:

- Language

- Emotion recognition

- Theory of mind

- Social skills.

The utility of technology and its wide implementation in autism has been recently recognized. Multimedia technology, applications, numerous blogs about computers and education of persons with autism and a remarkable increase in the number of relative publications and technology presentations per year affirm this trend. As far as emotion recognition and facial expressions are concerned, researchers have tried to employ CAT with people with autism to treat their deficits. More specifically, Golan et al (2006) used 'Mind Reading"' to train people with Asperger or high-functioning autism to recognize emotions in faces and voices through a taxonomic system of emotions and mental states. An extension of this software was also used by LaCava et al $(2007,2010)$. Another example is the 'Emotion Trainer' computer program which was used by Silver and Oakes (2001). This task includes five sections and children are asked to identify the emotion, describe the situation and indicate what (or whether) a picture corresponds to a certain situation or not. Similar to this approach, Moore et al (2005) employed a humanoid avatar simulating real life experiences and displaying four different emotions: sad, angry, happy and afraid. In three stages, the participants were asked to identify the emotion, predict it in different scenarios and select which situation could cause those emotions. The aforementioned studies emphasize that CAT programs can be a strong motivator for people with ASD, assisting them to improve their emotion recognition and develop new skills in a more accurate, interesting and efficient way. Furthermore, treatments based on such technologies are less expensive and accessible to broader parts of population. However, the superiority of CAT itself compared to more traditional- non-CAT approaches has not been undeniably demonstrated, so it is imperative that not pilot but large- scale studies with wider sample sizes and subgroups be conducted in the future [11].

Picard, R.W presents new affective technology that helps people with autism to communicate their emotions and maintain control over them during their social interactions. One of the most serious challenges autistic people face is the gap between what they really feel inwardly and display outwardly. This, in several cases, 
can result in high levels of frustration, stress, anger and even more problematic behaviors, like self- injury. At MIT Media Laboratory technology tools are developed to detect physiological data, like EDA (electrodermal activity), associated with emotion, cognition and attention, and assist people to realize their autonomic nervous system (ANS). For example, EDA is a good indicator of emotional arousal. The assessment, however, of these features is not simple, especially when the person overloads or experiences extreme pain and cannot manipulate the communication device. For this reason, it is important that unobtrusive wearable devices, adapted to individual needs and preferences, be invented. A device of this kind is EDA sensor; a wireless sensor packaged on the wrist, with disposable electrodes attached to the underside of a wristband. This sensor can be worn during activities for several weeks or months and can gather personalized physiological data. Tests checking the device's signal demonstrated that the wrist is a suitable site for EDA recording and that it is also important that factors, such as humidity or physical motion, be not taken into consideration. The author concludes that emotion communication technology utilizes information from everyday life enabling people with emotion interpretation problems to better understand and satisfy their needs [12].

\section{Conclusion}

This research once more has proved the correlation between ADHD and ASD. Researchers are still trying to understand and explain the cause for both condition and the impact of their combination. These disorders exhibit both difficulties in social and emotional skills. Handling the symptoms of ADHD can help handle the symptoms of ASD, too [Legg. J. Timothy, 2008]. According to Howlin P. "there are a variety of approaches that-although not resulting in 'cures' for autism (and ADHD as well) can result in improvement in many areas, including communication, social functioning and behavioral difficulties. For this purpose, we found that there are a lot of ICTs tools with realistic expectations that can help children with those specific developmental disorders to be more emotionally responsive as well as can help them to regulate their behavior deficits (the iSelf Control, the Chill Fish, the CAT, the EDA sensor). More research is still demanded to better understand the connection between ADHD and ASD and needed so that more intervention tools will be invented for the enhancement of both social and emotional skills [13, 14].

\section{$5 \quad$ References}

[1] Gadow, K. D., DeVincent, C. J., Pomeroy, J. (2006). ADHD symptom subtypes in children with pervasive developmental disorder. Journal of Autism and Developmental Disorders., 36(2):271-83 https://doi.org/10.1007/s10803-005-0060-3

[2] Harstad, E. (2017). I have heard that autism and ADHD are related. Is that true? UNDERSTOOD.ORG, from https://www.understood.org/en/learning-attentionissues/child-learning-disabilities/add-adhd/ive-heard-that-autism-and-adhd-are-related-isthat-true 
[3] Morin, A., (2014). The difference between ADHD and Autism. UNDERSTOOD.ORG, from https://www.understood.org/en/learning-attention-issues/child-learningdisabilities/add-adhd/the-difference-between-adhd-and-autism

[4] Bertin, M. (2016). Childhood Social Difficulties: The ADHD and Autism Relationship. huffingtonpost.com, from https://www.huffingtonpost.com/mark-bertin-md/childhoodsocial-difficulties-the-adhdautism-relationship_b_8389026.html

[5] Carpetner Rich, E., Loo, S. K., Yang, M., Dang, J., Smalley, S. L. (2009). Social functioning difficulties in ADHD: Association with PDD risk. Clinical Child Psychology and Psychiatry, 14(3): 329-344 https://doi.org/10.1177/1359104508100890

[6] Craig F., Margari F., Matera E., Simone M., Lamanna A., Margari L., (2015). Overlap between Autism Spectrum Disorders and Attention Deficit Hyperactivity Disorder: Searching for Distinctive/Common Clinical Features. Autism Res, https://www.ncbi.nlm.nih.gov/pmc/articles/PMC4654237/ https://doi.org/10.1002/aur.1449

[7] Taurines R, Schwenck C, Westerwald E, Sachse M, Siniatchkin M, et al. ADHD and autism: differential diagnosis or overlapping traits? A selective review. Attention Deficit and Hyperactivity Disorders. 2012; 4:115-139. [PubMed] https://doi.org/10.1007/s12402$\underline{012-0086-2}$

[8] Reiersen AM, Constantino JN, Volk HE. Todd RD. Autistic traits in a population-based ADHD twin sample. The Journal of Child Psychology and Psychiatry. 2007; 48:464472. [PubMed] https://doi.org/10.1111/j.1469-7610.2006.01720.x

[9] Ronald A, Simonoff E, Kuntsi J, Asherson P. Plomin R. Evidence for overlapping genetic influences on autistic and ADHD behaviours in a community twin sample. The Journal of Child Psychology and Psychiatry. 2008; 49:535-542. [PubMed] https://doi.org/10.111 1/j.1469-7610.2007.01857.x

[10] Schuck, S., Emmerson, N., Ziv, H., Collins, P., Arastoo, S., Warschauer, M., Crinella, F., Lakes, K. (2016). Designing an iPad App to Monitor and Improve Classroom Behavior for Children with ADHD: iSelfControl Feasibility and Pilot Studies. PLoS On, 11(10):e0164229. https://doi.org/10.1371/journal.pone.0164229

[11] Sonne, T., Møller Jensen, M. (2016). ChillFish: A Respiration Game for Children with ADHD. Conference: the TEI '16: Tenth International Conference https://doi.org/10.1 $\underline{145 / 2839462.2839480}$

[12] Sonne, T., Marshall. P., Müller, J., Obel, C., Grønbæk, K. (2016). A Follow-up Study of a Successful Assistive Technology for Children with ADHD and Their Families. In Proceedings of the The 15th International Conference on Interaction Design and Children (IDC '16). ACM, New York, NY, USA, 400-407. https://doi.org/10.1 $\underline{145 / 2930674.2930704}$

[13] Sonne, T., Marshall, P., Obel, C., Thomsen, P., Grønbæk, K. (2016). An assistive technology design framework for ADHD. In Proceedings of the 28th Australian Conference on Computer-Human Interaction (OzCHI '16). ACM, New York, NY, USA, 60-70. https://doi.org/10.1145/3010915.3010925

[14] Ploog, B. O., Scharf, A., Nelson, D., Brooks, P. J. (2013). Use of computer-assisted technologies (CAT) to enhance social, communicative, and language development in children with autism spectrum disorders. Journal of Autism and Developmental Disorders, 43(2):301-22. https://doi.org/10.1007/s10803-012-1571-3

[15] Picard, R. W. (2009). Future affective technology for autism and emotion communication. Philosophical Transactions of the Royal Society B: Biological Sciences, 364(1535), 3575-3584. https://doi.org/10.1098/rstb.2009.0143

[16] Legg, J. T, Holland, K, (April 25, 2018).The Relationship between ADHD and Autism. Healthline, 
[17] Howlin P., (2017). Evaluating psychological treatments for children with autism-spectrum disorders. Advances in psychiatric treatment (2010), vol. 16, 133-140 https://doi.org/1 $\underline{0.1192 / \text { apt.bp.109.006684 }}$

\section{Authors}

L. Bakola is a secondary-school teacher of Greek language. She is also a post graduate student in the programme "Expertise in ICT and Special Education Psychopedagogy of Integration" and a research associate at N.C.S.R. 'Demokritos', Institute of Informatics and Telecommunications, Net Media Lab, Agia Paraskevi, 15310 , Athens, Greece.

N. Rizos is a primary-school teacher. He is also a post graduate student in the programme "Expertise in ICT and Special Education - Psychopedagogy of Integration" and a research associate at N.C.S.R. 'Demokritos', Institute of Informatics and Telecommunications, Net Media Lab, Agia Paraskevi, 153 10, Athens, Greece

A. Drigas is a Research Director at N.C.S.R. 'Demokritos', Institute of Informatics and Telecommunications, Telecoms Lab - Net Media Lab, Agia Paraskevi, 153 10, Athens, Greece (e-mail: dr@iit.demokritos.gr).

Article submitted 2018-08-28. Resubmitted 2019-01-18. Final acceptance 2019-01-18. Final version published as submitted by the authors. 